\title{
Interaction Parameter Predicted by Pressure-Volume-Temperature Properties of Miscible Polymer Blends
}

\author{
Kee Su JeOn, Kookheon Char, ${ }^{\dagger}$ and Eugene $\mathrm{KIM}^{*, \dagger}$ \\ Department of Chemical Engineering, Seoul National University, \\ 56-1 Shinlimdong, Kwanakgu, Seoul 151-742, Korea \\ * Department of Applied Science, Hongik University, \\ 72-I Sangsoodong, Mapogu, Seoul 121-791, Korea,
}

(Received December 7, 1999)

\begin{abstract}
Expressions for Flory interaction parameter, $\chi_{\mathrm{sc}}$, were derived from the second derivative of the free energy of mixing by the following three equation of state theories: lattice fluid model of Sanchez and Lacombe [J. Phys. Chem., 80, 2352, 2568 (1976)], model of Flory, Orwoll, and Vrij [J. Am. Chem. Soc.. 86, 3507 (1964)], and modified cell model by Dee and Walsh [Macromolecules, 21, 815 (1988)]. In each case, composition dependence of the characteristic pressure parameter, $P^{*}$, was used to account for excess intermolecular interaction energy. The liquid state pressure-volume-temperature $(P V T)$ properties of both pure components and mixtures of polystyrene and tetramethylbisphenol-A polycarbonate were measured at the weight fraction of polystyrene of $1 / 3$ and $2 / 3$, which were accordingly analyzed. The absolute values of $\chi_{\mathrm{sc}}$ were always significantly larger than those obtained by small angle neutron scattering [H. Yang and J. M. O'Reilly, Mater. Res. Soc. Symp. Proc., 79, 129 (1987)] and diffusion [E. Kim et al., J. Polym. Sci., Polym. Phys. Ed., 33, 467 (1995)] measurements by a factor of $10^{2}$. The $P V T$ properties of blends misleadingly overestimate the enthalpic contribution, as also noted for the polystyrene (PS)/poly(vinyl methyl ether) mixture system by Ougizawa, Dee, and Walsh [Macromolecules, 24, 3834 (1991)].

KEY WORDS Polymer Blends / Interaction Parameter / Pressure-Volume-Temperature Properties / Equation of State Theories /
\end{abstract}

Some polymer pairs, the constituents of which do not form strong attractive interaction through hydrogen bonding or charge transfer, are miscible, ${ }^{8}$ while most high molecular weight polymer pairs are immiscible because of small gain in combinatorial entropy of mixing. The origins of miscibility of induced phase separation as the temperature is raised-lower critical solution temperature (LCST) phenomenon-have been extensibly studied. Two most comprehensive entropic contributions have been proposed. One is the effect caused by random mixture's finite compressibility which destabilizes the phase by heating, called equation of state (EOS) effect, ${ }^{1-4,7,9-12}$ and the other is the specific interaction between the components which gives rise to the preferred local alignment of unlike chains. ${ }^{9}$ EOS theories have been widely used to explain the thermodynamics of mixing of various polymer mixtures which do not exhibit strong inter-segmental interactions. Various quantitative EOS theories based on lattice model have been developed. Among well established and frequently used ones are the lattice fluid theory of Sanchez-Lacombe (SL), ${ }^{1,2}$ Flory, Owell, Vrij model (FOV), ${ }^{3}$ the modified cell model (MCM) ${ }^{4,7}$ While the hole model of Simha-Somcynsky ${ }^{11}$ is acknowledged as more flexible by introducing one additional parameter associated with available lattice sites, it was not used in the present study.

In most previous works, ${ }^{9,10,12}$ the characteristic parameters, $P^{*}, V^{*}$, and $T^{*}$ were obtained by fitting EOS to pressure-volume-temperature $(P V T)$ data of pure components. Equations for the equal chemical potential of the constituent chains (binodal condition) or equation for the destabilization criterion of the phase (spinodal condition) were used, and the characteristic parameter $\Delta P^{*}$, called bare interaction energy, was found. The

\footnotetext{
† To whom correspondence should be addressed.
}

theories have been applied along the region where the phase separation takes place, and it has not been well verified whether the intermolecular interaction in the single phase region is accounted for. Eichinger and Flory $^{13,14}$ already discussed the deviation of the calculated interaction parameter from experimental results in polymer solutions and introduced a correction parameter $Q_{12} \cdot{ }^{13}$ McMaster ${ }^{15}$ suggests a modified combining rules on mixing by introducing a nonlinear relation of the external degrees of freedom. Recently Shiomi et al. used the modified combining rules without adjustable parameter $Q_{12}$ and applied their scheme to polystyrene (PS)/poly(vinyl methyl ether) (PVME) blend, ${ }^{16}$ copolymer solutions, ${ }^{17}$ and copolymer blends. ${ }^{18,19}$ The spatial rearrangement of unlike chains caused by specific interaction ${ }^{9}$ or molecular association of the chains formed even in the absence of specific interaction ${ }^{20}$ may be an important factor for elucidating these extra entropic contributions to miscibility.

If miscibility is present without apparent specific interaction, EOS contribution may be expected to be dominant. Kim and Paul showed from the atomic charge calculations that the polymer mixture of PS and tetramethylbisphenol-A polycarbonate (TMPC) has relatively weak interaction, and they applied the equation of state theory (SL) to pure components' $P V T$ data and to the phase boundary of the mixtures. ${ }^{10}$ They claim that the Flory interaction parameter in the original zeroth order Flory-Huggins equation thus obtained is approximately in accord with the SANS results. Kim et al. ${ }^{6}$ claim from analysis based on the generalized LatticeFluid model by Sanchez and Balazs ${ }^{9}$ that the significant specific interaction is needed to explain the temperature dependence of $\chi_{\mathrm{sc}}$ in the same polymer mixtures.

This work derives the general expression for Flory-Huggins interaction parameter $\chi_{\mathrm{sc}}$ obtained from 
the second derivative of the free energy of mixing ${ }^{21}$ ("sc" stands for "scattering") by applying SL, FOV, and MCM, and investigates the intermolecular interaction contribution predicted by EOS formalism. Characteristic parameters were extracted from pure components and mixtures, and excess $\Delta P^{*}$ was obtained from the composition dependence of the $P^{*}$ by applying a conventional molecularly-motivated combining rule. $\chi_{\mathrm{sc}}$ was calculated and analyzed.

\section{EQUATION OF STATE THEORIES AND $\chi_{\text {sc }}$}

Differences in SL, FOV, and MCM theories originate from the definition of lattice, kind of ensemble used, configuration part in the partition function and inter-lattice interaction form. In SL, the size of the lattice is fixed and vacant sites exist for compressibility. The pressure ensemble is chosen and the theory of Guggenheim $^{22}$ is used. Interaction potential used in the theory was approximated to be proportional to $1 / V$. In FOV and MCM, the lattice size can change, while the vacant sites do not exist. The square well approximation $^{23}$ is used and partition function is defined from the canonical ensemble. For the potential, $1 / V$ is used in FOV and the Lenard-Jones 6-12 potential is used in MCM. The hexagonal lattice geometry was introduced in MCM, while the cubic lattice was used in FOV. Gibbs free energy of polymeric system, $G$, is found from partition function, $Z$.

$$
G=k T \ln Z
$$

Using following eq 2 for SL, and eq 3 for FOV and MCM, EOSs were derived.

$$
\begin{gathered}
\left.\frac{\partial \bar{G}}{\partial V}\right|_{\tilde{T}, \tilde{P}}=0 \\
P=\left.k T\left(\frac{\partial \ln Z}{\partial V}\right)\right|_{T}
\end{gathered}
$$

$\tilde{G}$ corresponds to reduced Gibbs free energy, $G / r N \varepsilon^{*}$, where $r$ is the number of segments (mers) per chain, $N$ is total number of chains, and $\varepsilon^{*}$ is the mean intermolecular energy per contact pair. The resulting EOSs are as follows.

For SL,

$$
\tilde{\rho}^{2}+\tilde{P}+\tilde{T}\left[\ln (1-\tilde{\rho})+\left(1-\frac{1}{r}\right) \tilde{\rho}\right]=0
$$

For FOV,

$$
\frac{\tilde{P} \tilde{V}}{\tilde{T}}=\frac{\tilde{V}^{1 / 3}}{\tilde{V}^{1 / 3}-1}-\frac{1}{\tilde{T} \tilde{V}}
$$

For MCM,

$$
\frac{\tilde{P} \tilde{V}}{\tilde{T}}=\frac{\tilde{V}^{1 / 3}}{\tilde{V}^{1 / 3}-q \cdot \gamma}-\frac{2}{\tilde{T}}\left(\frac{A}{\tilde{V}^{2}}-\frac{B}{\tilde{V}^{4}}\right)
$$

where $\tilde{P}, \tilde{T}, \tilde{\rho}$, and $\tilde{V}$ are reduced pressure, temperature, density, and specific volume $\left(\widetilde{P}=P / P^{*}, \quad \widetilde{T}=T / T^{*}\right.$, $\left.\tilde{\rho}=\rho / \rho^{*}, \tilde{V}=\tilde{V} / V^{*}=1 / \tilde{\rho}\right)$, respectively, $A=1.2045, B=$ $1.011, \gamma=1 / 2^{1 / 6}$ determined assuming that the cell lattice has a hexagonal close packed geometry, and empirical $q$ is $1.07 .^{4}$ EOSs for the mixtures were formally identical with those for the pure polymers.

To establish the partition functions of polymer mixtures relevant to each model, it was assumed that hard-core mer volumes are equal for all compositions. The conventional mixing rule was used and the hard core-pressure of the mixture was summed in such a way that $\Delta P^{*}$ could be estimated as follows, ${ }^{24}$

$$
P^{*}=\phi_{1} P_{1}^{*}+\phi_{2} P_{2}^{*}-\phi_{1} \theta_{2} \Delta P^{*}
$$

where $P_{\mathrm{i}}^{*}, \phi_{\mathrm{i}}$, and $\theta_{\mathrm{i}}$ are hard core-pressure, hard-core volume fraction and site fraction of component $i$, respectively. $\theta_{2}$ is identical to $\phi_{2}$ in $\mathrm{SL}$ and $\theta_{2}$ was approximated as $\phi_{2}$ in FOV and MCM. Gibbs free energy of mixing per lattice site, $\Delta g_{\mathbf{M}}$, is,

$$
\Delta g_{\mathrm{M}}=\Delta g_{\mathrm{c}}+\Delta g_{\mathrm{nc}}
$$

where $\Delta g_{\mathrm{c}}$ and $\Delta g_{\mathrm{nc}}$ correspond to the combinatorial entropy term and non-combinatorial free energy of mixing term, respectively. $\Delta g_{\mathrm{c}}$ is given by,

$$
\begin{aligned}
\Delta g_{\mathrm{c}} & =k T\left(\frac{\phi_{1}}{r_{1}} \ln \phi_{1}+\frac{\phi_{2}}{r_{2}} \ln \phi_{2}\right) \\
& =\frac{k T}{r N}\left(N_{1} \ln \phi_{1}+N_{2} \ln \phi_{2}\right)
\end{aligned}
$$

where $r_{\mathrm{i}}$ and $N_{\mathrm{i}}$ are the number of segments in a polymer chain and number of polymer chains of species $i$, respectively.

$\Delta g_{\mathrm{nc}}$ may be derived for each theory as follows.

For SL,

$$
\begin{aligned}
\Delta g_{\mathrm{nc}}= & V_{\mathrm{m}}^{*}\left[-\tilde{\rho}\left(\phi_{1} P_{1}^{*}+\phi_{2} P_{2}^{*}-\phi_{1} \phi_{2} \Delta P^{*}\right)\right. \\
& \left.+P \tilde{V}+\frac{R T}{V^{*}}\left(\frac{1-\tilde{\rho}}{\tilde{\rho}} \ln (1-\tilde{\rho})+\frac{\ln \tilde{\rho}}{r}\right)\right]
\end{aligned}
$$

where $V_{\mathrm{m}}^{*}$ is the core mer volume. Properties without subscripts are those for mixtures.

For FOV,

$$
\begin{aligned}
\Delta g_{\mathrm{nc}}= & V_{\mathrm{m}}^{*}\left[\phi_{1} P_{1}^{*}\left(\frac{1}{\tilde{V}_{1}}-\frac{1}{\tilde{V}}\right)+\phi_{2} P_{2}^{*}\left(\frac{1}{\tilde{V}_{2}}-\frac{1}{\widetilde{V}}\right)\right. \\
& +3 \phi_{1} P_{1}^{*} T \ln \frac{\tilde{V}^{1 / 3}-1}{\tilde{V}^{1 / 3}-1} \\
& \left.+3 \phi_{2} P_{2}^{*} \tilde{T}_{2} \ln \frac{\tilde{V}_{2}^{1 / 3}-1}{\tilde{V}^{1 / 3}-1}+\frac{\phi_{1} \phi_{2} \Delta P^{*}}{\tilde{V}}\right]
\end{aligned}
$$

For MCM,

$$
\begin{aligned}
\Delta g_{\mathrm{nc}}= & V_{\mathrm{m}}^{*}\left[\phi_{1} P_{1}^{*}\left\{A\left(\frac{1}{\tilde{V}_{1}^{2}}-\frac{1}{\tilde{V}^{2}}\right)-\frac{B}{2}\left(\frac{1}{\tilde{V}_{1}^{4}}-\frac{1}{\tilde{V}^{4}}\right)\right\}\right. \\
& +\phi_{12} P_{2}^{*}\left\{A\left(\frac{1}{\tilde{V}_{2}^{2}}-\frac{1}{\tilde{V}^{2}}\right)-\frac{B}{2}\left(\frac{1}{\tilde{V}_{2}^{4}}-\frac{1}{\tilde{V}^{4}}\right)\right\} \\
& +3 \phi_{1} P_{1}^{*} \tilde{T}_{1} \ln \frac{\tilde{V}_{1}^{1 / 3}-q \cdot \gamma}{\tilde{V}^{1 / 3}-q \cdot \gamma} \\
& +3 \phi_{2} P_{2}^{*} \tilde{T}_{2} \ln \frac{\tilde{V}_{2}^{1 / 3}-q \cdot \gamma}{\tilde{V}^{1 / 3}-q \cdot \gamma}
\end{aligned}
$$




$$
\left.+\phi_{1} \phi_{2} \Delta P^{*}\left(\frac{A}{\tilde{V}^{2}}-\frac{B}{2 \tilde{V}^{4}}\right)\right]
$$

In scattering and diffusion experiments, the second concentration derivative of free energy of mixing is directly probed, and is equivalent to the inverse of the equilibrium structure factor where the wave vector approaches to $0, S^{-1}(0)$.

$$
S^{-1}(0)=\frac{d^{2}\left(\Delta g_{\mathrm{M}} / k T\right)}{d \phi_{1}^{2}}=\frac{1}{r_{1} \phi_{1}}+\frac{1}{r_{2} \phi_{2}}-2 \chi_{\mathrm{sc}}
$$

When the Flory interaction parameter has composition dependence, the original zeroth order form differs from $\chi_{\text {sc }}$. Sanchez has rigorously derived the relations between interaction parameters defined depending on the experimental methods of probing mixtures' free energy. ${ }^{21}$ The second order interaction parameter $\chi_{\text {sc }}$ can be obtained from the following relation,

$$
\frac{\chi_{\mathrm{sc}}}{V_{\mathrm{ref}}}=\frac{1}{2 R T}\left\{\frac{R T}{V_{\mathrm{ref}}}\left(\frac{1}{r_{1} \phi_{1}}+\frac{1}{r_{2} \phi_{2}}\right)-\frac{d^{2} \Delta G_{\mathrm{M}}}{d \phi_{1}^{2}}\right\}
$$

where $\Delta G_{\mathrm{M}}$ is Gibbs free energy of mixing per molar mer volume and $V_{\text {ref }}$ is the reference molar volume for which $\chi_{\mathrm{sc}}$ is defined ( $\chi_{\mathrm{sc}}$ is divided by $V_{\mathrm{ref}} / R T$ to become the interaction energy density).

$$
\Delta G_{\mathrm{M}}=\frac{\Delta g_{\mathrm{M}} \cdot N_{\mathrm{Avo}}}{V_{\mathrm{ref}}}
$$

$N_{\text {Avo }}$ is Avogadro's number and $V_{\text {ref }}$ is taken as $V_{\mathrm{mm}}^{*}$, the molar core mer volume $\left(V_{\mathrm{m}}^{*} \cdot N_{\mathrm{Avo}}\right)$ in EOS analysis. In FOV and MCM theories, $\chi_{\mathrm{sc}} / V_{\text {ref }}$ may be directly obtained without requiring the value of $V_{\mathrm{mm}}^{*}$. In the SL model, the molecular weight of the mer is calculated using $R T^{*} \rho^{*} / P^{*}$, from which $V_{\mathrm{mm}}^{*}$ is estimated. In this case, the value of $V_{\mathrm{mm}}^{*}$ ranged from 13 to $16 \mathrm{~cm}^{3} \mathrm{~mol}^{-1}$ in PS/TMPC mixtures. The hypothetical common monomer volume for estimating $\chi_{\mathrm{sc}}$ in $\mathrm{SANS}^{5}$ and diffu$\operatorname{sion}^{6}$ measurements was taken as the geometric mean of volumes of structural repeating units of PS and TMPC, about $175 \mathrm{~cm}^{3} \mathrm{~mol}^{-1}$ (thermal expansion of $V_{\text {ref }}$ was also taken into account). Equation 14 basically includes all terms in the second concentration derivative of $\Delta G_{\mathrm{M}}$ except for combinatorial entropy terms.

The second derivative of the Gibbs free energy with respect to composition is given by,

$$
\frac{d^{2} \Delta g_{\mathrm{M}}}{d \phi_{1}^{2}}=\Delta g_{\mathrm{M} \phi \phi}-\frac{\left(\Delta g_{\mathrm{M} \tilde{\rho} \phi}\right)^{2}}{\Delta g_{\mathrm{M} \tilde{\rho} \tilde{\rho}}}
$$

where subscript $\phi$ and $\tilde{\rho}$ indicate partial derivatives with respect to $\phi_{1}$ and $\tilde{\rho}$. The expression for $\chi_{\mathrm{sc}}$ in SL, FOV, and MCM is obtained from eq 14-16, written for each theory as,

$$
\underset{\text { ref }}{\chi_{\mathrm{sc}}}=\frac{1}{2 R T}\left\{a+\frac{(b T+c)^{2}}{d}-\right\}
$$

For SL,

$$
\begin{aligned}
& a=2 \tilde{\rho} \Delta P^{*} \\
& b=R\left\{\frac{1}{\tilde{\rho}}\left(\begin{array}{c}
1 \\
r_{1} V_{\mathrm{mm}, 1}^{*}
\end{array}-\frac{1}{r_{2} V_{\mathrm{mm}, 2}^{*}}\right)\right.
\end{aligned}
$$

$$
\begin{aligned}
& \left.\quad-\left[\frac{\ln (1-\tilde{\rho})}{\tilde{\rho}^{2}}+\frac{1}{\tilde{\rho}}\right]\left[\begin{array}{cc}
\frac{1}{V_{\mathrm{mm} .1}^{*}}-V_{\mathrm{mm}, 2}^{*}
\end{array}\right]\right\} \\
& c=P_{2}^{*}-P_{1}^{*}+\left(\phi_{2}-\phi_{1}\right) \Delta P^{*} \\
& d=\frac{R}{V_{\mathrm{mm}}^{*}}\left[\frac{2 \ln (1-\tilde{\rho})}{\tilde{\rho}^{3}}+\frac{1}{\tilde{\rho}^{2}(1-\tilde{\rho})}+\frac{\left(1-\frac{1}{r}\right)}{\tilde{\rho}^{2}}\right]
\end{aligned}
$$

For FOV,

$$
\begin{aligned}
& a=\frac{2{ }^{s_{1}} \tilde{\rho} \Delta P^{*}}{\left(1+\left(\frac{s_{1}}{s_{2}}-1\right) \phi_{1}\right)^{3}} \\
& b=\left(\frac{P_{1}^{*}}{T_{1}^{*}}-\frac{P_{2}^{*}}{T_{2}^{*}}\right)\left(\frac{\tilde{\rho}^{-4 / 3}}{\tilde{\rho}^{-1 / 3}-1}\right) \\
& c=-P_{1}^{*}+P_{2}^{*}+\left(\begin{array}{c}
1-2 \phi_{1}-\left(\frac{s_{1}}{s_{2}}-1\right) \phi_{1}^{2} \\
\left(1+\left(\frac{s_{1}}{s_{2}}-1\right) \phi_{1}\right)^{2}
\end{array}\right) \Delta P^{*} \\
& d=\left(\frac{\phi_{1} P_{1}^{*}}{T_{1}^{*}}+\frac{\phi_{2} P_{2}^{*}}{T_{2}^{*}}\right)\left(\frac{-\tilde{\rho}^{-2 / 3}+\frac{4 \tilde{\rho}^{-1 / 3}}{3}}{\left(\tilde{\rho}^{2 / 3}-\tilde{\rho}\right)^{2}}\right)
\end{aligned}
$$

where $s_{\mathrm{i}}$ is the number of contact sites per unit core volume of species $i$.

For MCM,

$$
\begin{aligned}
& a=\frac{2 \frac{s_{1}}{s_{2}}\left(A \tilde{\rho}^{2}-\frac{B}{2} \tilde{\rho}^{4}\right) \Delta P^{*}}{\left(1+\left(\frac{s_{1}}{s_{2}}-1\right) \phi_{1}\right)^{3}} \\
& b=\left(\frac{P_{1}^{*}}{T_{1}^{*}}-\frac{P_{2}^{*}}{T_{2}^{*}}\right)\left(\frac{\tilde{\rho}^{-4 / 3}}{\tilde{\rho}^{-1 / 3}-q r}\right) \\
& c=\left(P_{2}^{*}-P_{1}^{*}\right)\left(2 A \tilde{\rho}-2 B \tilde{\rho}^{3}\right) \\
& +\left(\frac{1-2 \phi_{1}-\left(\frac{s_{1}}{s_{2}}-1\right) \phi_{1}^{2}}{\left(1+\left(\frac{s_{1}}{s_{2}}-1\right) \phi_{1}\right)^{2}}\right) \Delta P^{*} \\
& d=\left(\frac{\phi_{1} P_{1}^{*}}{T_{1}^{*}}+\frac{\phi_{2} P_{2}^{*}}{T_{2}^{*}}\right)\left(\begin{array}{c}
-\tilde{\rho}^{-2 / 3}+q r \frac{4 \tilde{\rho}^{-1 / 3}}{3} \\
\frac{\left(\tilde{\rho}^{2 / 3}-q r \tilde{\rho}\right)^{2}}{2}
\end{array}\right)
\end{aligned}
$$

the ratio of $s_{\mathrm{PS}}$ to $s_{\mathrm{TMPC}}$ was obtained by estimating the surface areas of PS and TMPC per $V^{*}$ using Bondi's method, ${ }^{25}$ and was 0.857 for MCM and 0.876 for FOV.

\section{EXPERIMENTAL}

Weight-average molecular weights and polydispersity indices of PS were 253000 and 2.0, and those of TMPC were 42000 and 2.9 , respectively. The $P V T$ relation was measured as follows for pure components and mixtures at PS weight fractions $w$ of $0,1 / 3,2 / 3$, and 1. First, 

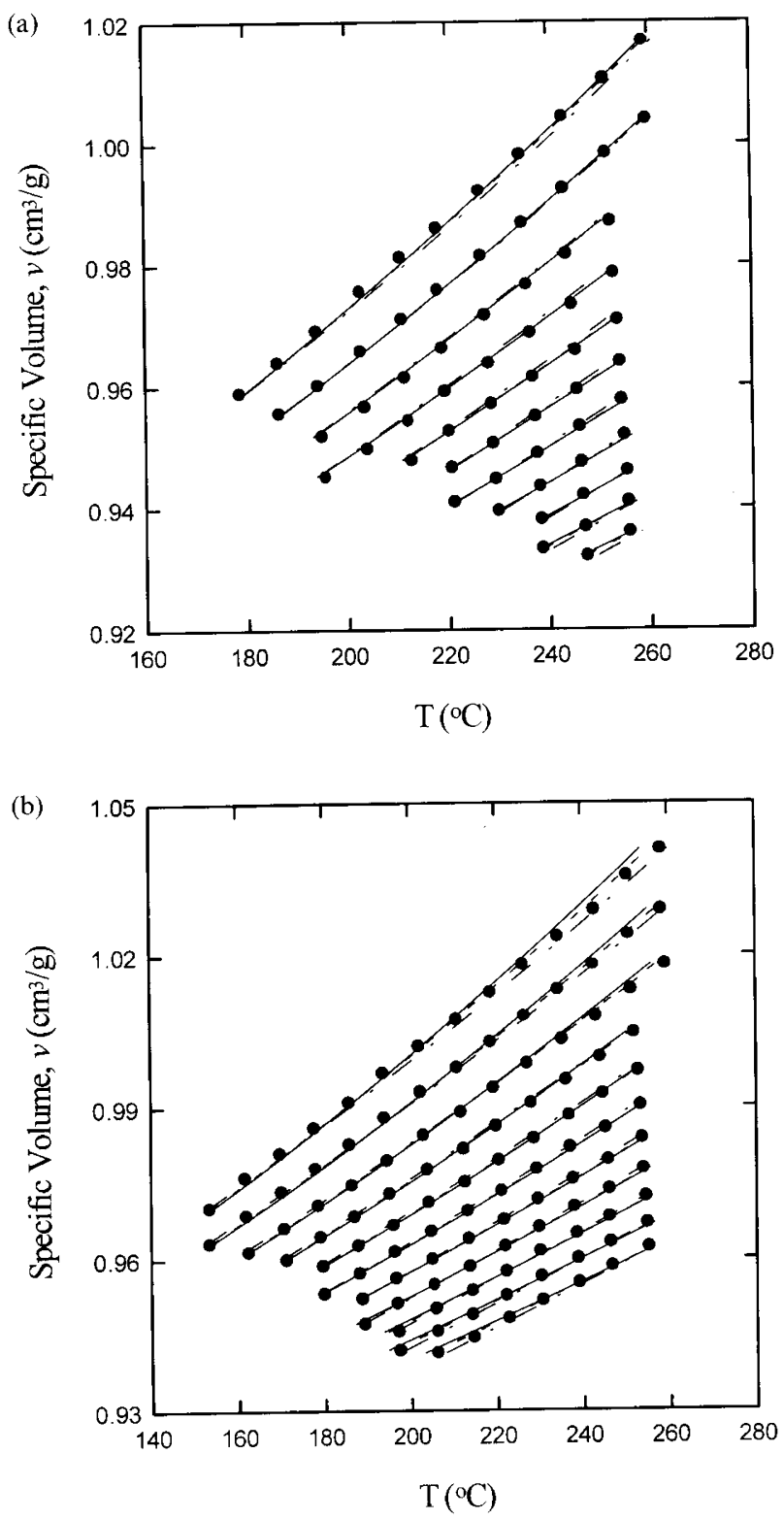

Figure 1. Dots are $P V T$ data of PS/TMPC mixtures from 0 to $100 \mathrm{MPa}$ by $10 \mathrm{MPa}$ at two PS weight fractions, $w$ : (a) at $w=1 / 3$ and (b) at $w=2 / 3$. Isobar lines are the fits to the three equations of state: SL (broken-dotted lines), FOV (dotted lines) and MCM (solid lines).

densities were measured at $25^{\circ} \mathrm{C}$ at atmospheric pressure using an autopycrometer (Micromeritics). Changes in density as a function of temperature (up to $\mathrm{ca} .280{ }^{\circ} \mathrm{C}$ by $8-10^{\circ} \mathrm{C}$ increment) and pressure (up to $200 \mathrm{MPa}$ by $10 \mathrm{MPa}$ increment) were measured using a $P V T$ apparatus. The absolute accuracy of the device is $10^{-3}-2 \times 10^{-3} \mathrm{~cm}^{3} \mathrm{~g}^{-1}$. Volume changes as small as $10^{-4}-2 \times 10^{-4} \mathrm{~cm}^{3} \mathrm{~g}^{-1}$ could be resolved. The temperature range for fitting was from the glass transition temperatures $\left(T_{\mathrm{g}}\right.$ ) to $260^{\circ} \mathrm{C}$ above which phase separation took place at $w$ of $1 / 3$ and $2 / 3$. The pressure range actually used in the melt $P V T$ analysis became $0-100 \mathrm{MPa}$ except for the pure TMPC because all data above $70 \mathrm{MPa}$ fell into the glassy region. The details of the procedure are fully described elsewhere. ${ }^{26}$ To obtain characteristic parameters for each EOS, we carried out a nonlinear least square fit of each EOS by minimizing the sum of the square of error divided by the number of $P V T$ data

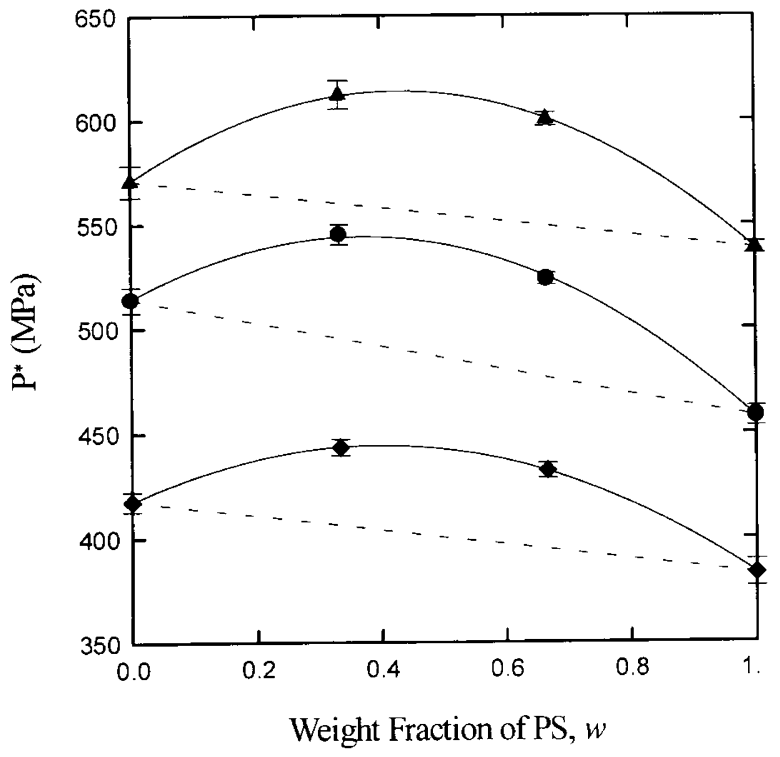

Figure 2. Characteristic pressures, $P^{*}$, in PS/TMPC mixtures as a function of PS weight fraction $w$. $[\boldsymbol{\bullet}, \boldsymbol{\Delta}]$ correspond to values obtained by SL, FOV, and MCM, respectively. Solid lines represent the values obtained by fitting the points for each theory to eq 7 , and the dotted lines represent additive values obtained using $P^{*}$ of pure components. Error bars represent $S E\left(P^{*}\right)$ shown in Table II.

$(N)$ subtracted by the number of estimated parameters (3).

$$
S^{2}=\frac{\sum_{i}\left(P_{i, \mathrm{data}}-P_{i, \mathrm{fit}}\right)^{2}}{N-3}
$$

Where $P_{i, \text { data }}$ and $P_{i, \text { fit }}$ are pressures measured and predicted by relevant EOSs at a given $i(V, T)$ for the system, respectively. Figure 1 illustrates the $P V T$ data above $T_{\mathrm{g}}$ (dots) and estimations (lines) fitted using three EOS theories (a) at $w=1 / 3$ and (b) at $w=2 / 3$. The data of pure PS and TMPC gave similar fits. Table I shows the corresponding characteristic parameters $\left(P^{*}, \rho^{*}, T^{*}\right)$, $S^{2}$, and standard error(SE) for each EOS at each $w$. The way to estimate $S E$ in each charateristic parameter using a given set of $P V T$ data is explained in APPENDIX, assuming that error occurs only in the fitting procedure. There was no apparent difference in goodness of data fitting to these three EOSs.

\section{ESTIMATION OF $\chi_{\mathrm{sc}}$}

$P *_{\mathrm{S}}$ at 4 compositions obtained from SL, FOV, and MCM manifest positive deviation from additive lines as shown in Figure 2. The size of the error bar in Figure 2 is $\operatorname{SE}\left(P^{*}\right)$ in Table I. The interaction parameter $\Delta P^{*}$ was computed by eq 7 . These results are given in Table II. Ougizawa et $a l^{7}$ studied the $P V T$ properties of pure components and mixtures of PS and PVME by applying FOV and MCM and $\Delta P^{*}$ is shown together for comparison. The cloud points and pure components' $P V T$ measurements were made in the PS/TMPC system by $\mathrm{Kim}$ and $\mathrm{Paul}^{10}$ and in the PS/PVME system by Walsh et al. ${ }^{12} \mathrm{Kim}$ and Paul applied SL and Walsh et al. applied FOV and cell model. $\Delta P^{*}$ are also listed in Table II. The values obtained from the $P V T$ data of mixtures were by far larger than the ones obtained by 
Table I. Characteristic parameters of pure components and mixtures and goodness of fit (refer to APPENDix)

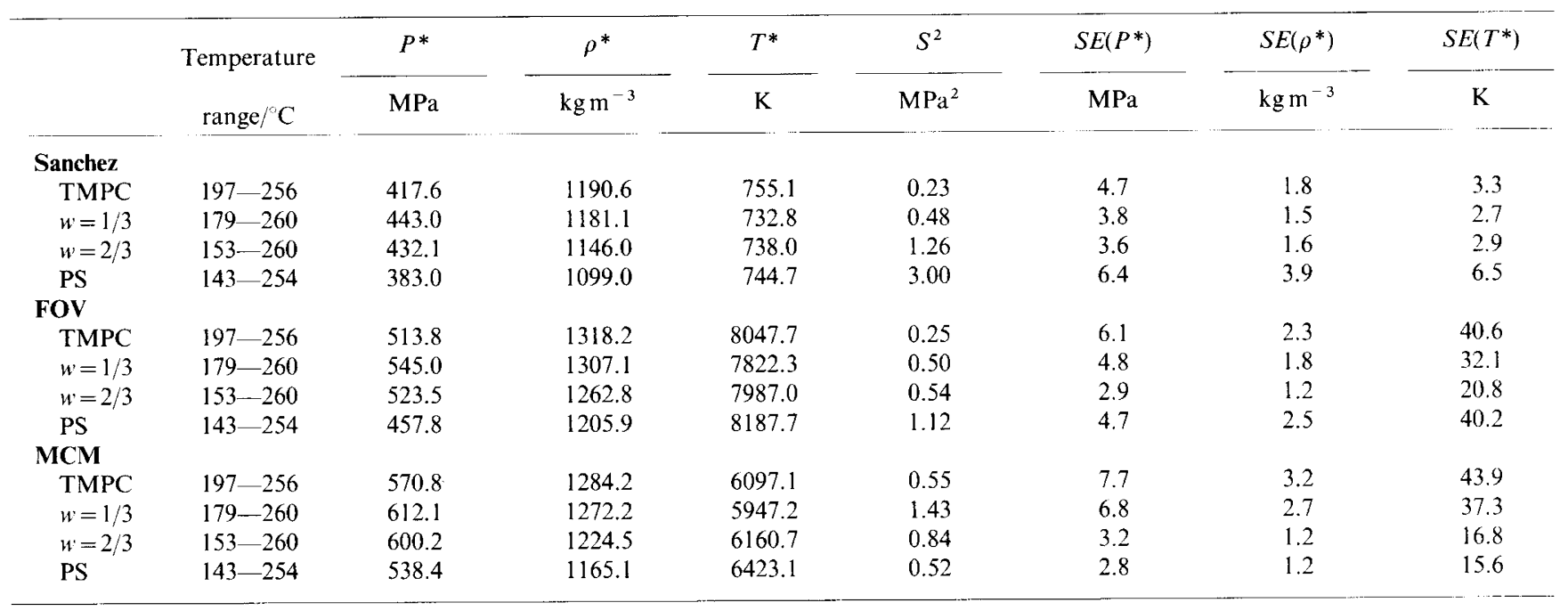

Table II. Interaction parameters $\Delta P^{*}(\mathrm{MPa})$

\begin{tabular}{|c|c|c|c|c|c|c|}
\hline & \multicolumn{3}{|c|}{ Blends $P V T$} & \multicolumn{3}{|c|}{ Cloud point measurements } \\
\hline & SL & FOV & $\mathrm{MCM}$ & SL & FOV & $\mathrm{CM}^{\mathrm{a}}$ \\
\hline $\begin{array}{l}\text { PS/TMPC } \\
\text { PS/PVME }\end{array}$ & -170.5 & $\begin{array}{l}-223.0 \\
-150^{7}\end{array}$ & $\begin{array}{l}-235.2 \\
-300^{7}\end{array}$ & $\begin{array}{c}-0.17^{10} \\
-\end{array}$ & $-\overline{1.78^{1}}$ & $-\overline{1.76^{12}}$ \\
\hline
\end{tabular}

${ }^{\mathrm{a}}$ Cell model.

cloud point measurement by about a factor of $10^{3}$. The large negative value of $\Delta P^{*}$ was also obtained by Ougizawa et al. from PS/PVME blend $P V T$ data. The analysis based on temperature dependence $\Delta P^{*}$ also predicts that phase separation ${ }^{6}$ cannot occur before the liquid-gas transition temperature. Ougizawa et al. pointed out that the discrepancy in phase separation behavior may be partly explained by allowing $\Delta P^{*}$ to have temperature dependence ${ }^{7}\left(\Delta P^{*}\right.$ has to become smaller as temperature is raised). If it was due to the extra entropic effect such as specific interaction, caused by the spatial rearrangement of unlike chains, this interaction would decrease at higher temperature due to thermal agitation. However subsequent analysis which thus introduces the temperature dependence to $\Delta P^{*}$ led to a false interpretation of $P V T$ data. ${ }^{7}$. The magnitude of $\Delta P^{*}$ predicted from the $P V T$ data turned out to be too large at any temperature except for temperatures close to the phase separation temperatures.

$\chi_{\text {sc }}$ parameters were computed for each composition using equations $17-20 . \chi_{\mathrm{sc}} \mathrm{s}$ were divided by the corresponding reference molar volumes for comparison of values obtained by different experimental methods. Figure 3 shows the temperature dependence of $\chi_{\mathrm{sc}} / V_{\text {ref }}$ obtained from each EOS. The results obtained from light scattering $^{10}$ and diffusion ${ }^{6}$ are shown together. The major observation was that absolute $\chi_{\mathrm{sc}} \mathrm{s}$ obtained from the characteristic pressure $P^{*}$ of both pure components and mixtures were larger than those obtained from diffusion ${ }^{6}$ or SANS $^{5}$ measurements by about a factor of $10^{2} \cdot \chi_{\mathrm{sc}} \mathrm{s}$ obtained from $\Delta P^{*}$ in Table II obtained from the cloud point measurements ${ }^{10,12}$ were significantly smaller than those obtained separately. Therefore neither the results obtained from blend $P V T$ data nor by $P V T$

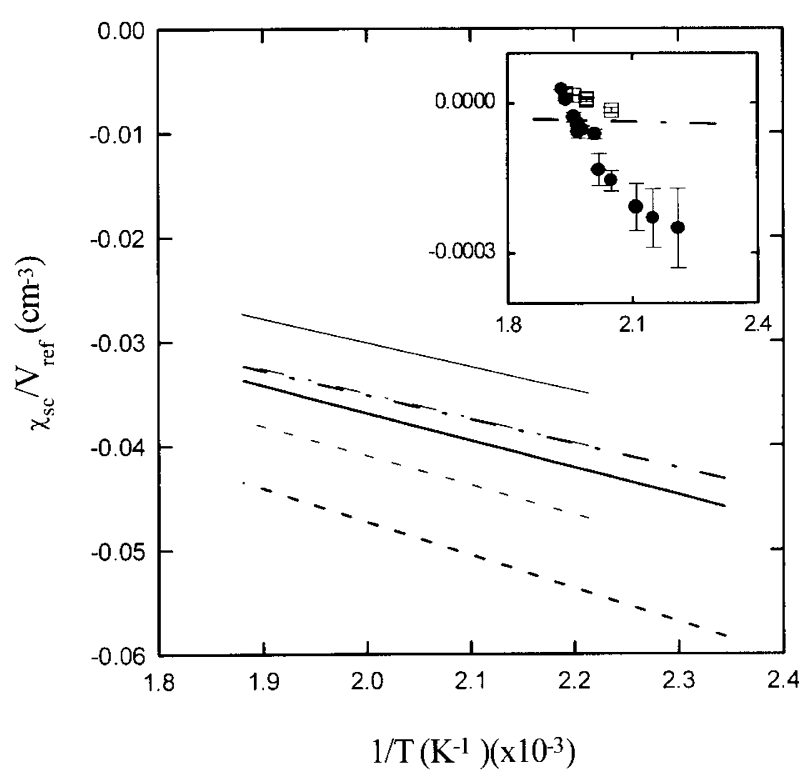

Figure 3. Temperature dependence of $\chi_{\mathrm{sc}} / V_{\mathrm{ref}}$ calculated at PS weight fraction $w=1 / 3$ (thinner broken-dotted line), $2 / 3$ (thicker broken-dotted line) for SL, $w=1 / 3$ (thinner dotted line), 2/3 (thicker dotted line) for FOV and $w=1 / 3$ (thinner solid line), 2/3 (thicker solid line) for MCM. Since the broken-dotted lines for SL nearly overlap, they are represented as one line. In the inset (note the difference in scale), the broken-dotted line represents the values using $\Delta P^{*}$ obtained by fitting cloud points to $\mathrm{SL},{ }^{10}$ where no difference was observed at $w=1 / 3$ and at $w=2 / 3$. [ $\square, \bigcirc$ correspond to the values obtained from the diffusion experiments ${ }^{5}$ at $w=0.25$ and 0.5 , respectively. For the definition of $V_{\text {ref }}$ refer to the text.

data of pure components and cloud point data properly explain the observed thermodynamics of PS/TMPC mixture system in one phase region.

To assess the entropic and enthalpic contributions to non-combinatorial free energy of mixing, computed in the present EOS frameworks, $\chi_{\mathrm{sc}}$ was devided as following.

$$
\begin{gathered}
\chi_{\mathrm{H}}=-T \partial \chi_{\mathrm{sc}} / \partial T \\
\chi_{\mathrm{s}}=\partial\left(T \chi_{\mathrm{sc}}\right) / \partial T
\end{gathered}
$$

The results are shown in Figures 4(a) and 4(b). Enthalpic contribution $\left(\chi_{\mathrm{H}}\right)$ in $\chi_{\mathrm{sc}}$ is dominant due to the negatively large $\Delta P^{*}$. Entropic contribution $\left(\chi_{\mathrm{s}}\right)$ should become 
(a)
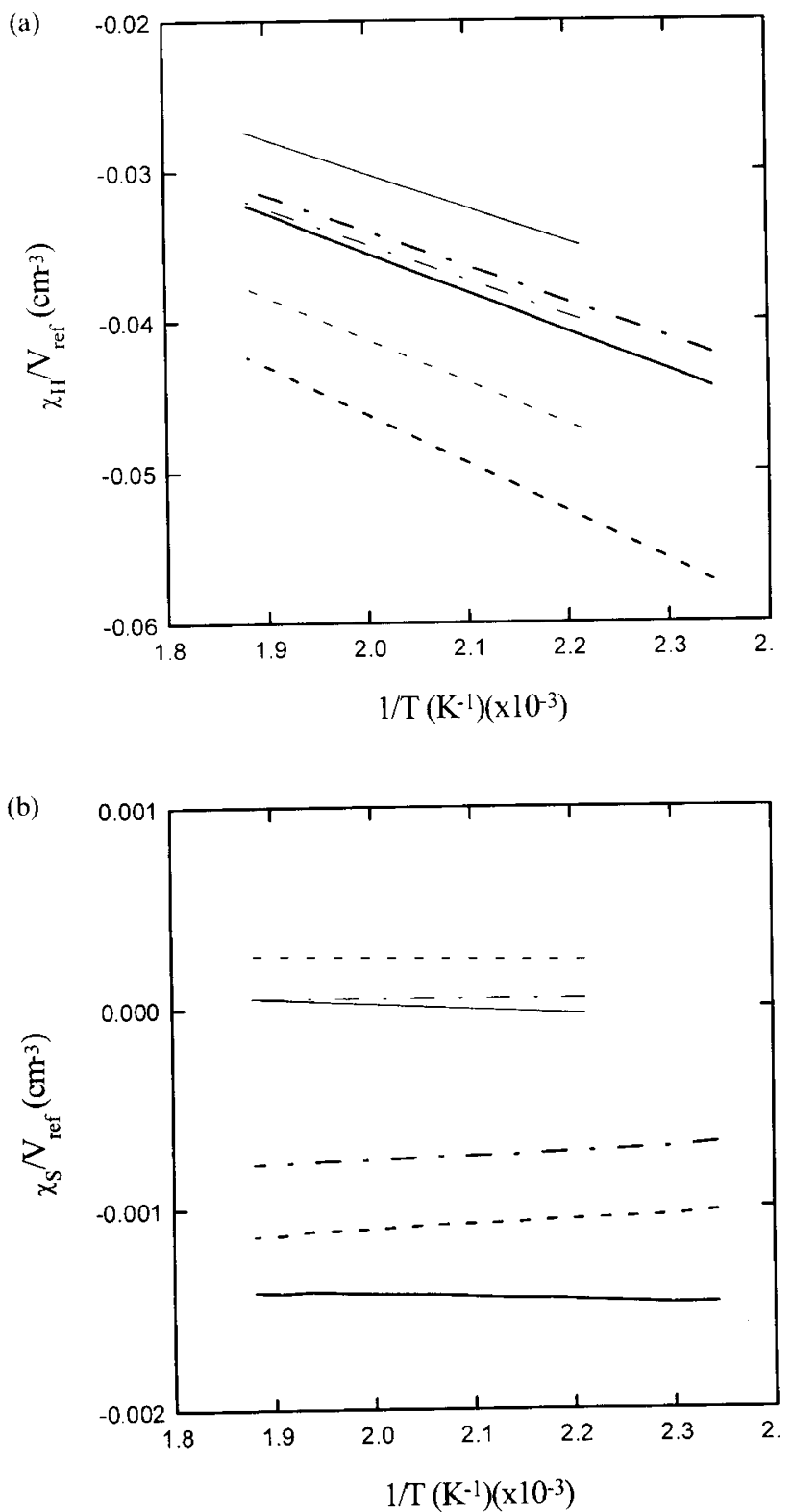

Figure 4. (a). Enthalpic part of the $\chi_{\mathrm{sc}} / V_{\text {ref }}$ obtained from eq 22. Same line symbols were used as in Figure 3. (b). Entropic part of the $\chi_{\mathrm{sc}} / V_{\text {ref }}$ obtained from eq 23. Same line symbols were used as in Figure 3.

larger and more positive as temperature increases if thermally induced LCST is driven by the entropic penalty which originates from the compressibility effect. The contribution of $\chi_{s}$ to $\chi_{s c}$ was only minor as shown in Figure 4(b). The slight difference in temperature dependence of $\chi_{\mathrm{s}}$ at different compositions seems due to very small difference in compressibility between pure PS and TMPC, which acts to suppress the compressibility effect.

In diffusion and SANS experiments, larger negative $\chi_{\mathrm{sc}}$ was observed at larger $w^{5,6}$ (the blend becomes more miscible as composition becomes PS-rich). $\chi_{\text {sc }}$ as a function of composition obtained at $T_{\mathrm{g}}+45^{\circ} \mathrm{C}$ using eq $17-20$ is shown in Figure 5, and is compared with other data obtained from other experimental methods. Even though the absolute magnitude deviates significantly, $\chi_{\mathrm{sc}}$ obtained in this study showed a similar trend to what were found in the other data. More composition de-

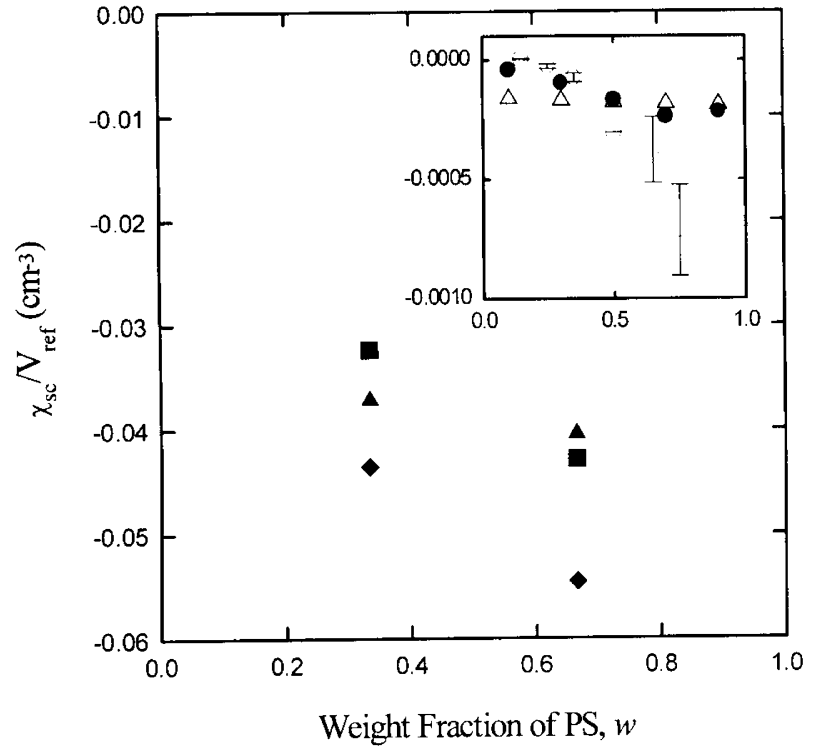

Figure 5. $\chi_{\mathrm{sc}} / V_{\text {ref }}$ was calculated as a function of PS weight fraction $w$ for three theories at $T_{\mathrm{g}}+45^{\circ} \mathrm{C}$. The symbols $[\boldsymbol{\nabla}, \boldsymbol{\Delta}, \bullet]$ correspond to the values obtained from MCM, SL, and FOV, respectively. In the inset (note the difference in scale), the values obtained from SANS experiments ${ }^{5}(O)$ and the diffusion experiment $(O)$ at $T_{\mathrm{g}}+45^{\circ} \mathrm{C}^{6}$ were represented as functions of $w$. Symbol $\triangle$ in the inset represents $\chi_{\mathrm{sc}} / V_{\text {ref }}$ values at $30 \mathrm{C}$ obtained by fitting the cloud points to the spinodal condition using SL theory. ${ }^{10}$ The temperature at which SANS data were obtained is not clear here. ${ }^{6}$

pendence was observed in FOV and MCM than in SL, which arises from the fact that change in lattice size induces change in the number of contact sites between the mers.

\section{CONCLUSIONS}

Equations for the interaction parameter in the second concentration derivative of the free energy of mixing expression, $\chi_{\mathrm{sc}}$, were derived using various EOSs. $P V T$ properties of pure components and mixtures of PS and TMPC were measured and the corresponding EOS theories were applied to find the characteristic parameters, $P^{*}, V^{*}$, and $T^{*} . \Delta P^{*}$ was obtained from the composition dependence of the $P^{*}$, from which the scattering Flory-Huggins interaction parameter $\chi_{\text {sc }}$ was computed. $\Delta P^{*}$ and $\chi_{\mathrm{sc}}$ were negative and significantly larger than those obtained by other techniques. Similar results have been previously reported in PS and PVME mixtures. Thermodynamic data based on $P V T$ properties must be carefully interpreted in evaluating the noncombinatorial free energy contribution on mixing.

Acknowledgments. This paper was supported by NON DIRECTED RESEARCH FUND, Korea Research Foundation, 1996. EK is especially grateful to Professors Namhyun Kim and Heungsun Park for helpful discussion in making the Appendix for computing SE. We also thank the reviewers for useful comments.

\section{REFERENCES AND NOTES}

1. I. C. Sanchez and R. H. Lacombe, J. Phys. Chem., 80, 2352(1976).

2. I. C. Sanchez and R. H. Lacombe, J. Phys. Chem., 80, 2568 (1976). 
3. P. J. Flory, R. A. Orwoll, and A. Vrij, J. Am. Chem. Soc., 86, 3507 (1964).

4. G. T. Dee and D. J. Walsh, Macromolecules, 21, 815 (1988).

5. H. Yang and J. M. O'Reilly, Mater. Res. Soc. Symp. Proc., 79, 129 (1987).

6. E. Kim, E. J. Kramer, J. O. Osby, and D. J. Walsh, J. Polym. Sci., Polym. Phys. Ed., 33, 467 (1995)

7. T. Ougizawa, G. T. Dee, and D. J. Walsh, Macromolecules, 24, 3834 (1991)

8. J. E. Mark, Ed., "Physical Properties of Polymers Handbook (AIP Series in Polymers and Complex Materials)," American Institute of Physics, New York, N.Y., 1996, Chapter 19.

9. I. C. Sanchez and A. C. Balazes, Macromolecules, 22, 2324 (1989).

10. C. K. Kim and D. R. Paul, Polymer, 33, 1630 (1992).

11. T. Somcynsky and R. Simha, Macromolecules, 2, 343 (1969).

12. D. J. Walsh, G. T. Dee, J. L. Halary, J. M. Ubiche, M. Millequant, J. Lesec, and L. Monnerie, Macromolecules, 22, 3395 (1989).

13. B. E. Eichinger and P. J. Flory, Trans. Faraday Soc., 64, 2035 (1968).

14. B. E. Eichinger and P. J. Flory, Trans. Faraday Soc., 64, 2066 (1968); H. Höcker, G. J. Blake and P. J. Flory, ibid., 67, 2251 (1971); P. J. Flory and H. Höcher, ibid., 67, 2258 (1971)

15. Lee P. McMaster, Macromolecules, 6, 760 (1973).

16. T. Shiomi, F. Hamada, T. Nasako, K. Yoneda, K. Imai, and A. Nakajima, Macromolecules, 23, 229 (1990).

17. T. Shiomi, H. Ishimatsu, T. Eguchi, and K. Imai, Macromolecules, 23, 4970 (1990)

18. T. Shiomi, T. Eguchi, H. Ishimatsu, and K. Imai, Macromolecules, 23, $4978(1990)$

19. T. Sato, M. Tohyama, M. Suzuki, T. Shiomi, and K. Imai, Macromolecules, 29, 8231 (1996)

20. G. C. Reichart, W. W. Graessley, R. A. Register, R. Krishnamoorti, and D. J. Lohse, Macromolecules, 30, 3036 (1997); D. W. Tomlin, and C. M. Roland, ibid., 25, 2994 (1992).

21. I. C. Sanchez, Polymer, 30, 471 (1989).

22. E. A. Guggenheim, "Applications of Statistical Mechanics," Oxford University Press, London, 1966, Chapters 4 and 7.

23. I. Prigogine, H. Traffeniers, and V. Mathot, J. Chem. Phys., 26, 751 (1957).

24. T. Ougizawa and T. Inoue, in "Elastomer Technology Handbook," N. P. Cheremisinoff, Ed., CRC Press, Boca Raton, FL, 1993, Chapter 19.

25. A. Bondi, J. Phys. Chem., 68, 441 (1964).

26. P. Zoller and H. H. Hoehn, J. Polym. Sci., Polym. Phys. Ed., 20, 1385 (1982).

\section{APPENDIX}

The way to estimate $S E$ in $P^{*}, \rho^{*}$, and $T^{*}$

Let $\theta$ be $\left(P^{*}, \rho^{*}, T^{*}\right)$ the vector notation of the characteristic parameters, and $\varepsilon_{j}$ be the error in fitting the $j$-th data $P_{j, \text { data }}$ to $P_{j, \text { fit. }}$

$$
P_{j, \mathrm{data}}=P_{j, \mathrm{fit}}(\rho, T, \theta)+\varepsilon_{j}
$$

We introduced $S^{2}$ in eq 21 which can be rewritten as:

$$
S^{2}=\frac{\sum_{i} \varepsilon_{i}^{2}}{N-3}
$$

Smoothly varying function $P_{j, \text { fit }}(\rho, T, \underline{0})$ was linearized around $\hat{\theta}=\left(\hat{P}^{*}, \hat{\rho}^{*}, \hat{T}^{*}\right)$ as follows, where hat $\left(^{\wedge}\right)$ represents the fitted estimate.

$$
\begin{array}{r}
P_{j, \mathrm{fit}}(\rho, T, \underline{\theta}) \approx P_{j, \mathrm{fit}}(\rho, T, \underline{\hat{\theta}})+\left.\frac{\partial P_{j, \mathrm{fit}}}{\partial P^{*}}\right|_{\underline{\theta}=\underline{\theta}}\left(P^{*}-\hat{P}^{*}\right) \\
\quad+\left.\frac{\partial P_{j, \mathrm{fit}}}{\partial \rho^{*}}\right|_{\underline{\theta}=\underline{\theta}}\left(\rho^{*}-\hat{\rho}^{*}\right)+\left.\frac{\partial P_{j, \mathrm{fit}}}{\partial T^{*}}\right|_{\underline{\theta}=\underline{\sigma}}\left(T^{*}-\hat{T}^{*}\right)
\end{array}
$$

$\varepsilon_{j}$ may be estimated as follows:

$$
\begin{aligned}
\hat{\varepsilon}_{j}= & P_{j, \mathrm{data}}-P_{j, \mathrm{fit}}(\rho, T, \hat{\theta})=\left.\frac{\partial P_{j, \mathrm{fit}}}{\partial P^{*}}\right|_{\underline{\theta}=\underline{\theta}}\left(P^{*}-\hat{P}^{*}\right) \\
& +\left.\frac{\partial P_{j, \mathrm{fit}}}{\partial P^{*}}\right|_{\theta=\underline{\theta}}\left(\rho^{*}-\hat{\rho}^{*}\right)+\left.\frac{\partial P_{j, \mathrm{fit}}}{\partial T^{*}}\right|_{\theta=\underline{\theta}}\left(T^{*}-\hat{T}^{*}\right)
\end{aligned}
$$

Finally SE is obtained from the following relations, where Var stands for the asymptotic variance-covariance matrix when $N$ is sufficiently large.

$\operatorname{Var}(\underline{\hat{\theta}}) \approx\left(W^{T} W\right)^{-1} \hat{S}^{2} \quad$ where $\quad \hat{S}^{2}=\frac{\sum_{i} \hat{\varepsilon}_{i}^{2}}{N-3}$

$W=\left[\begin{array}{lll}\left.\frac{\partial P_{1, \mathrm{fit}}}{\partial P^{*}}\right|_{\underline{\theta}=\underline{\theta}} & \left.\frac{\partial P_{1, \mathrm{fit}}}{\partial \rho^{*}}\right|_{\underline{\theta}=\underline{\theta}} & \left.\frac{\partial P_{1, \mathrm{fit}}}{\partial T^{*}}\right|_{\underline{\theta}=\underline{\theta}} \\ \left.\left.\left.\frac{\partial P_{2, \mathrm{fit}}}{\partial P^{*}}\right|_{\underline{\theta}=\underline{\theta}} \frac{\partial P_{2, \mathrm{fit}}}{\partial \rho^{*}}\right|_{\underline{\theta}=\underline{\theta}} \frac{\partial P_{2, \mathrm{fit}}}{\partial T^{*}}\right|_{\underline{\theta}=\underline{\theta}} \\ \vdots \\ \left.\left.\left.\frac{\partial P_{N, \mathrm{fit}}}{\partial P^{*}}\right|_{\underline{\theta}=\underline{\hat{\theta}}} \frac{\partial P_{N, \mathrm{fit}}}{\partial \rho^{*}}\right|_{\underline{\theta}=\underline{\theta}} \frac{\partial P_{N, \mathrm{fit}}}{\partial T^{*}}\right|_{\underline{\theta}=\underline{\theta}}\end{array}\right]$

$V a r_{i j}$ is an (i-th, $j$-th) element of Var:

$$
S E\left(\hat{\theta}_{i}\right)=\sqrt{\operatorname{Var}}{ }_{i i}(\hat{\theta}), \quad i=1,2,3
$$

i.e., $S E\left(\hat{P}^{*}\right), S E\left(\hat{\rho}^{*}\right)$, and $S E\left(\hat{T}^{*}\right)$ are $S E\left(\hat{\theta}_{1}\right), S E\left(\hat{\theta}_{2}\right)$, and $S E\left(\hat{\theta}_{3}\right)$, respectively. 